\title{
Classification of Some Special Types Ruled Surfaces in Simply Isotropic 3-Space
}

\author{
Murat Kemal Karacan, Dae Won Yoon, and Nural Yuksel
}

\begin{abstract}
In this paper, we classify two types ruled surfaces in the three dimensional simply isotropic space $\mathbb{I}_{3}^{1}$ under the condition $\Delta \mathbf{x}_{i}=\lambda_{i} \mathbf{x}_{i}$ where $\Delta$ is the Laplace operator with respect to the first fundamental form and $\lambda$ is a real number. We also give explicit forms of these surfaces.
\end{abstract}

AMS Subject Classification (2000).

Keywords. Simply isotropic space, ruled surfaces, laplace operator.

\section{Introduction}

Let $\mathbf{x}: \mathbf{M} \rightarrow \mathbb{E}^{m}$ be an isometric immersion of a connected $n$-dimensional manifold in the $m$-dimensional Euclidean space $\mathbb{E}^{m}$. Denote by $\mathbf{H}$ and $\Delta$ the mean curvature and the Laplacian of $\mathbf{M}$ with respect to the Riemannian metric on $\mathbf{M}$ induced from that of $\mathbb{E}^{m}$, respectively. Takahashi ([17]) proved that the submanifolds in $\mathbb{E}^{m}$ satisfying $\Delta \mathbf{x}=\lambda \mathbf{x}$, that is, all coordinate functions are eigenfunctions of the Laplacian with the same eigenvalue $\lambda \in \mathbb{R}$, are either the minimal submanifolds of $\mathbb{E}^{m}$ or the minimal submanifolds of hypersphere $\mathbb{S}^{m-1}$ in $\mathbb{E}^{m}$.

As an extension of Takahashi theorem, in [8] Garay studied hypersurfaces in $\mathbb{E}^{m}$ whose coordinate functions are eigenfunctions of the Laplacian, but not 
necessarily associated to the same eigenvalue. He considered hypersurfaces in $\mathbb{E}^{m}$ satisfying the condition $\Delta \mathbf{x}=\mathbf{A x}$, where $\mathbf{A} \in$ Mat $(m, \mathbb{R})$ is an $m \times m$ diagonal matrix, and proved that such hypersurfaces are minimal $(\mathbf{H}=0)$ in $\mathbb{E}^{m}$ and open pieces of either round hyperspheres or generalized right spherical cylinders. Related to this, Dillen, Pas and Verstraelen ([6]) investigated surfaces in $\mathbb{E}^{3}$ whose immersions satisfy the condition $\Delta \mathbf{x}=\mathbf{A x}+\mathbf{B}$, where $\mathbf{A} \in \operatorname{Mat}(3, \mathbb{R})$ is a $3 \times 3$-real matrix and $\mathbf{B} \in \mathbb{R}^{3}$.

The notion of an isometric immersion $\mathbf{x}$ is naturally extended to smooth functions on submanifolds of Euclidean space or pseudo-Euclidean space. The most natural one of them is the Gauss map of the submanifold. In particular, if the submanifold is a hypersurface, the Gauss map can be identified with the unit normal vector field to it. Dillen, Pas and Verstraelen ([7]) studied surfaces of revolution in the three dimensional Euclidean space $\mathbb{E}^{3}$ such that its Gauss map $\mathbf{G}$ satisfies the condition $\Delta \mathbf{G}=\mathbf{A G}$, where $\mathbf{A} \in$ Mat $(3, \mathbb{R})$. Baikoussis and Verstraelen $([3])$ studied the helicoidal surfaces in $\mathbb{E}^{3}$. Yoon $([19,20])$ classified the surfaces of revolution and the translation surfaces in the 3-dimensional Galilean space and pseudo-Galilean 3-space under the condition $\Delta \mathbf{x}^{i}=\lambda^{i} x^{i}$ and $\Delta \mathbf{r}_{i}=\lambda_{i} \mathbf{r}_{i}$, where $\lambda^{i} \in \mathbb{R}$. Karacan and Yoon $([10,11])$ classified translation surfaces and helicoidal surfaces in the threedimensional simply isotropic space $\mathbb{I}_{3}^{1}$.

Kamenarović ([9]) studied the natural geometry of ruled surfaces and defined equations for the three types ruled surfaces in simply isotropic space $\mathbb{I}_{3}^{1}$. Sipus and Divjak ([15]) studied some mappings of skew ruled surfaces in simply isotropic space which preserve the generators.

The main purpose of this paper is to complete classification of special non-developable ruled surfaces of Type 3 and Type 4 defined by W.Vogel in the three dimensional simply isotropic space $\mathbb{I}_{3}^{1}$ in terms of the position vector field and the Laplacian operator.

\section{Preliminaries}

Motions and metric Isotropic geometry is based on the following group $\mathbf{G}_{6}$ of affine transformations $(x, y, z) \rightarrow\left(x^{\prime}, y^{\prime}, z^{\prime}\right)$ in $\mathbb{R}^{3}$,

$$
\begin{aligned}
& x^{\prime}=a+x \cos \theta-y \sin \theta \\
& y^{\prime}=b+x \sin \theta+y \cos \theta \\
& z^{\prime}=c++c_{1} x+c_{2} y+z,
\end{aligned}
$$


where $a, b, c, c_{1}, c_{2}, \theta \in R$. Such affine transformations are called isotropic congruence transformations or isotropic motions. We see that isotropic motions appear as Euclidean motions (a translation and a rotation) in the projection onto the $x y$-plane the result of this projection, $P=(x, y, z) \rightarrow$ $P^{\prime}=(x, y, 0)$ is called the "top view" ([11]). Hence, an isotropic motion is composed of a Euclidean motion in the $x y$-plane and an affine shear transformation in the $z$-direction.

On the other hand, the isotropic distance of two points $P=\left(x_{1}, y_{1}, z_{1}\right)$ and $Q=\left(x_{2}, y_{2}, z_{2}\right)$ is defined as the Euclidean distance of the top views, i.e.,

$$
d(P, Q)_{i}=\sqrt{\left(x_{1}-x_{2}\right)^{2}+\left(y_{1}-y_{2}\right)^{2}} .
$$

Let $X=\left(x_{1}, y_{1}, z_{1}\right)$ and $Y=\left(x_{2}, y_{2}, z_{2}\right)$ be vectors in $\mathbb{I}_{3}^{1}$. The isotropic inner product of $X$ and $Y$ is defined by

$$
\langle X, Y\rangle_{i}=\left\{\begin{array}{cc}
z_{1} z_{2}, & \text { if } x_{i}=y_{i}=0 \\
x_{1} x_{2}+y_{1} y_{2}, & \text { if otherwise. }
\end{array}\right.
$$

We call a vector of the form $X=(0,0, z)$ in $\mathbb{I}_{3}^{1}$ an isotropic vector, and a non-isotropic vector otherwise. Consider a $C^{r}$-surface $\mathbf{M}, 1 \leq r$, in $\mathbb{I}_{3}^{1}$ parameterized by

$$
\mathbf{x}(u, v)=(x(u, v), y(u, v), z(u, v)) .
$$

A surface $\mathbf{M}$ immersed in $\mathbb{I}_{3}^{1}$ is called admissible if it has no isotropic tangent planes. We restrict our framework to admissible regular surfaces ([21]).

For such a surface, the coefficients $E, F, G$ of its first fundamental form are calculated with respect to the induced metric and the coefficients $L, M, N$ of the second fundamental form, with respect to the normal vector field of a surface which is always completely isotropic. The first and the second fundamental form of $\mathbf{M}$ are defined by

$$
\begin{aligned}
I & =E d u^{2}+F d u d v+G d v^{2}, \\
I I & =L d u^{2}+M d u d v+N d v^{2},
\end{aligned}
$$

where

$$
\begin{aligned}
E & =\left\langle\mathbf{x}_{u}, \mathbf{x}_{u}\right\rangle_{i}, F=\left\langle\mathbf{x}_{u}, \mathbf{x}_{v}\right\rangle_{i}, G=\left\langle\mathbf{x}_{v}, \mathbf{x}_{v}\right\rangle_{i} \\
L & =\frac{\operatorname{det}\left(\mathbf{x}_{u}, \mathbf{x}_{v}, \mathbf{x}_{u u}\right)}{\sqrt{E G-F^{2}}}, M=\frac{\operatorname{det}\left(\mathbf{x}_{u}, \mathbf{x}_{v}, \mathbf{x}_{u v}\right)}{\sqrt{E G-F^{2}}}, N=\frac{\operatorname{det}\left(\mathbf{x}_{u}, \mathbf{x}_{v}, \mathbf{x}_{v v}\right)}{\sqrt{E G-F^{2}}} .
\end{aligned}
$$

Since $E G-F^{2}>0$, for the function in the denominator we often put $W^{2}=E G-F^{2}$. The isotropic unit normal vector field is given by $\mathbf{U}=(0,0,1)$. 
The isotropic curvature $\mathbf{K}$ and the isotropic mean curvature $\mathbf{H}$ are defined by

$$
\mathbf{K}=\frac{L N-M^{2}}{E G-F^{2}}, \quad 2 \mathbf{H}=\frac{E N-2 F M+G L}{E G-F^{2}} .
$$

The surface $\mathbf{M}$ is said to be isotropic flat (resp. isotropic minimal), if $\mathbf{K}$ (resp. H) vanishes $([1,10,12,16,21])$.

It is well known in terms of local coordinates $\{u, v\}$ of $\mathbf{M}$ the Laplacian operators $\Delta$ of the first fundamental form on $\mathbf{M}$ are defined by $([2,4])$

$$
\Delta \mathbf{x}=-\frac{1}{\sqrt{E G-F^{2}}}\left[\frac{\partial}{\partial u}\left(\frac{G \mathbf{x}_{u}-F \mathbf{x}_{v}}{\sqrt{E G-F^{2}}}\right)-\frac{\partial}{\partial v}\left(\frac{F \mathbf{x}_{u}-E \mathbf{x}_{v}}{\sqrt{E G-F^{2}}}\right)\right]
$$

\section{$3 \quad$ Ruled Surfaces in $\mathbb{I}_{3}^{1}$}

Let $\mathbf{M}$ be ruled surface in $\mathbb{I}_{3}^{1}$ given by the parametrization

$$
\begin{aligned}
\mathbf{x} & : \quad I \times \mathbb{R} \rightarrow \mathbb{I}_{3}^{1} \\
(u, v) & \rightarrow \quad \mathbf{x}(u, v)=\alpha(u)+v \beta(u) .
\end{aligned}
$$

We call the base curve $\alpha$ and the director curve $\beta$, where $\alpha$ is a differentiable curve parametrized by its arc length, i.e., $\left\langle\alpha^{\prime}, \alpha^{\prime}\right\rangle_{i}=1$ and $\langle\beta, \beta\rangle_{i}=1$. The curve $\beta$ is orthogonal to the tangent vector field $T_{\alpha}$ of the base curve $\alpha$, i.e., $\left\langle\beta^{\prime}, T_{\alpha}\right\rangle=0$. First of all, we consider non isotropic plane curves $\alpha$ and $\beta$ parametrized by $\alpha(u)=(u, 0, f(u))$ and $\beta(u)=(0,1, g(u))$. Then the surface $\mathbf{M}$ is parametrized by

$$
\mathbf{x}(u, v)=(u, v, f(u)+v g(u))
$$

We consider isotropic curve $\alpha=(0,0, f(u))$ and non isotropic space curve $\beta$ parametrized by $\beta(u)=(\cos u, \sin u, g(u))$, where $\langle\beta, \beta\rangle_{i}=1$. Then the surface $\mathbf{M}$ is parametrized by

$$
\mathbf{x}(u, v)=(v \cos u, v \sin u, f(u)+v g(u))
$$

The functions $f$ and $g$ are smooth functions of one variable. We call the surfaces given by (3.2) and (3.3) as ruled surfaces of Type 3 and Type 4 in the three dimensional simply isotropic space $\mathbb{I}_{3}^{1}$, respectively $([12,18])$. 


\section{Ruled Surfaces of Type 3 Satisfying $\Delta \mathrm{x}_{i}=\lambda_{i} \mathrm{x}_{i}$}

In this section, we classify the ruled surface of Type 3 in $\mathbb{I}_{3}^{1}$ satisfying the equation

$$
\Delta \mathbf{x}_{i}=\lambda_{i} \mathbf{x}_{i}
$$

where $\lambda_{i} \in \mathbb{R}, i=1,2,3$ and

$$
\Delta \mathbf{x}=\left(\Delta \mathbf{x}_{1}, \Delta \mathbf{x}_{2}, \Delta \mathbf{x}_{3}\right),
$$

where

$$
\mathbf{x}_{1}=u, \mathbf{x}_{2}=v, \mathbf{x}_{3}=f(u)+v g(u) .
$$

For the ruled surface given by (3.2), the coefficients of the first and second fundamental form are

$$
\begin{gathered}
E=1, F=0, G=1, \\
L=0, M=g^{\prime}, N=f^{\prime \prime}+v g^{\prime \prime},
\end{gathered}
$$

respectively. The Gaussian curvature $\mathbf{K}$ and the mean curvature $\mathbf{H}$ are

$$
\mathbf{K}=-g^{\prime^{2}}, \quad \mathbf{H}=\frac{f^{\prime \prime}+v g^{\prime \prime}}{2}
$$

respectively.

Proposition 4.1. The Ruled surface given by (3.2) in the three dimensional simply isotropic space $\mathbb{I}_{3}^{1}$ are isotropic flat or developable $(\mathbf{K}=0)$, iff $g(u)=$ $c_{1}$ for constant $c_{1}$.

Suppose that the surface has non zero the Gaussian curvature, so $g^{\prime}(u) \neq$ 0 . By a straightforward computation, the Laplacian operator on $\mathbf{M}$ with the help of (3.2) and (2.7) turns out to be

$$
\Delta \mathbf{x}_{i}=\left(0,0,-f^{\prime \prime}(u)-v g^{\prime \prime}(u)\right) .
$$

Suppose that $\mathbf{M}$ satisfies (4.1). Then from (4.5), we have

$$
\left(f^{\prime \prime}(u)+v g^{\prime \prime}(u)\right)=-\lambda(f(u)+v g(u)),
$$

where $\lambda \in \mathbb{R}$. This means that $\mathbf{M}$ is at most of 1-type. First of all, we assume that $\mathbf{M}$ satisfies the condition $\Delta \mathbf{x}_{i}=0$. We call a surface satisfying that condition a harmonic surface or isotropic minimal. In this case, we get from (4.6)

$$
f^{\prime \prime}(u)+v g^{\prime \prime}(u)=0 .
$$


The general solutions of the equation (4.7) with respect to $f$ and $g$ are given by

$$
\begin{aligned}
& f(u)=c_{1} u+c_{2}-v g(u), \\
& g(u)=c_{3} u+c_{4}-\frac{f(u)}{v},
\end{aligned}
$$

where $c_{i} \in \mathbb{R}$. Here, the functions $f$ and $g$ are related. Based on the selection of the function $f(u)$, it is possible to obtain other form of the function $g(u)$. For example, if we choose $f(u)=\ln u$, we have $g(u)=c_{3} u+c_{4}-\frac{\ln u}{v}$. In this case, $\mathbf{M}$ is parametrized by

$$
\mathbf{x}(u, v)=\left(u, v, \ln u+v\left(c_{3} u+c_{4}-\frac{\ln u}{v}\right)\right) .
$$

Theorem 4.2. Let $\mathbf{M}$ be a ruled surface given by (3.2) in $\mathbb{I}_{3}^{1}$. If $\mathbf{M}$ is harmonic or isotropic minimal, then it is congruent to an open part of the surface

$$
\mathbf{x}(u, v)=\left(u, v, f(u)+v\left(c_{3} u+c_{4}-\frac{f(u)}{v}\right)\right) .
$$

If $\lambda \neq 0$, from (4.6), we have

$$
\left.\left(f^{\prime \prime}(u)+\lambda f(u)\right)+v\left(g^{\prime \prime}(u)+\lambda g(u)\right)=0\right) .
$$

This equations are second order linear differential equations with constant coefficients. We discuss two cases according to constant $\lambda$.

Case 1: Let $\lambda>0$, from (4.9), we obtain

$$
\begin{aligned}
& f(u)=c_{1} \cos u \sqrt{\lambda}+c_{2} \sin u \sqrt{\lambda}-v g(u), \\
& g(u)=c_{3} \cos u \sqrt{\lambda}+c_{4} \sin u \sqrt{\lambda}-\frac{f(u)}{v},
\end{aligned}
$$

where $\lambda, c_{i} \neq 0 \in \mathbb{R}$. Here, the functions $f$ and $g$ are related. Based on the selection of the function $f(u)$ or $g(u)$, it is possible to obtain other form of the function $g(u)$ or $f(u)$. For example, if we choose $f(u)=\ln u$, In this case, $\mathbf{M}$ is parametrized by

$$
\mathbf{x}(u, v)=\left(u, v, \ln u+v\left(c_{3} \cos u \sqrt{\lambda}+c_{4} \sin u \sqrt{\lambda}-\frac{\ln u}{v}\right)\right) .
$$

Case 2: Let $\lambda<0$, from (4.9), we obtain

$$
\begin{aligned}
& f(u)=c_{1} e^{u \sqrt{\lambda}}+c_{2} e^{-u \sqrt{\lambda}}-v g(u), \\
& g(u)=c_{3} e^{u \sqrt{\lambda}}+c_{4} e^{-u \sqrt{\lambda}}-\frac{f(u)}{v},
\end{aligned}
$$


where $c_{i} \in \mathbb{R}$. If we choose $f(u)=\ln u$, we have

$$
\mathbf{x}(u, v)=\left(u, v, \ln u+v\left(c_{3} e^{u \sqrt{\lambda}}+c_{4} e^{-u \sqrt{\lambda}}-\frac{\ln u}{v}\right)\right) .
$$

Theorem 4.3. Let $\mathbf{M}$ be a non harmonic ruled surface given by (3.2) in the three dimensional simply isotropic space $\mathbb{I}_{3}^{1}$. If the surface $\mathbf{M}$ satisfies the condition $\Delta \mathbf{x}_{i}=\lambda_{i} \mathbf{x}_{i}$, where $\lambda_{i} \in \mathbb{R}, i=1,2,3$, then it is congruent to an open part of the following surfaces

$$
\mathbf{x}(u, v)=\left(u, v, f(u)+v\left(c_{3} \cos u \sqrt{\lambda}+c_{4} \sin u \sqrt{\lambda}-\frac{f(u)}{v}\right)\right)
$$

and

$$
\mathbf{x}(u, v)=\left(u, v, f(u)+v\left(c_{3} e^{u \sqrt{\lambda}}+c_{4} e^{-u \sqrt{\lambda}}-\frac{f(u)}{v}\right)\right) .
$$

\section{Ruled Surfaces of Type 4 Satisfying $\Delta \mathrm{x}_{i}=\lambda_{i} \mathrm{x}_{i}$}

In this section, we classify the ruled surface of Type 4 in $\mathbb{I}_{3}^{1}$ satisfying the equation

$$
\Delta \mathbf{x}_{i}=\lambda_{i} \mathbf{x}_{i}
$$

where $\lambda_{i} \in \mathbb{R}, i=1,2,3$ and

$$
\Delta \mathbf{x}=\left(\Delta \mathbf{x}_{1}, \Delta \mathbf{x}_{2}, \Delta \mathbf{x}_{3}\right)
$$

where

$$
\mathbf{x}_{1}=v \cos u, \mathbf{x}_{2}=v \sin u, \mathbf{x}_{3}=f(u)+v g(u) .
$$

For the ruled surface given by (3.3), the coefficients of the first and second fundamental form are

$$
\begin{gathered}
E=v^{2}, F=0, G=1, \\
L=-f^{\prime \prime}-v g^{\prime \prime}-v g, M=g^{\prime}, N=0,
\end{gathered}
$$

respectively. The Gaussian curvature $\mathbf{K}$ and the mean curvature $\mathbf{H}$ are

$$
\mathbf{K}=-\frac{g^{\prime^{2}}}{v^{2}}, \quad \mathbf{H}=-\frac{f^{\prime \prime}+v\left(g^{\prime \prime}+g\right)}{2 v^{2}},
$$

where $v \neq 0$,respectively. 
Proposition 5.1. The Ruled surface given by (3.3) in the three dimensional simply isotropic space $\mathbb{I}_{3}^{1}$ are isotropic flat or developable $(\mathbf{K}=0)$, iff $g(u)=$ $c_{1}$ for constant $c_{1}$.

Suppose that the surface has non zero the Gaussian curvature, so $g^{\prime}(u) \neq$ 0 . By a straightforward computation, the Laplacian operator on $\mathbf{M}$ with the help of (3.3) and (2.7) turns out to be

$$
\Delta \mathbf{x}_{i}=\left(0,0,-\frac{f^{\prime \prime}+v\left(g^{\prime \prime}+g\right)}{v^{2}}\right) .
$$

Suppose that $\mathbf{M}$ satisfies (5.1). Then from (5.5), we have

$$
\frac{f^{\prime \prime}+v\left(g^{\prime \prime}+g\right)}{v^{2}}=-\lambda(f+v g)
$$

where $\lambda \in \mathbb{R}$. This means that $\mathbf{M}$ is at most of 1-type. First of all, we assume that $\mathbf{M}$ satisfies the condition $\Delta \mathbf{x}_{i}=0$. We call a surface satisfying that condition a harmonic surface or isotropic minimal. In this case, we get from $(5.6)$

$$
f^{\prime \prime}(u)+v\left(g^{\prime \prime}(u)+g(u)\right)=0
$$

The general solutions of the equation (5.7) with respect to $f$ and $g$ are given by

$$
\begin{aligned}
f(u)= & c_{1} u+c_{2}+\int_{1}^{u}\left(-v \int_{1}^{z}\left(g^{\prime \prime}(s)+g(s)\right) d s\right) d z \\
g(u)= & c_{3} \cos u+c_{4} \sin u+\cos u\left(\int_{1}^{u} \frac{f^{\prime \prime}(x) \sin x}{v} d x\right) \\
& -\sin u\left(\int_{1}^{u} \frac{f^{\prime \prime}(y) \sin y}{v} d y\right)
\end{aligned}
$$

where $c_{i} \in \mathbb{R}$. Here, the functions $f$ and $g$ are related. Based on the selection of the function $f(u)$ or $g(u)$, it is possible to obtain other form of the function $g(u)$ or $f(u)$. For example, if we choose $f(u)=e^{u}$, we have

$$
g(u)=\frac{-e^{u}+e \cos (1-u)+2 c_{3} v \cos u+2 c_{4} v \sin u+e^{u} \sin 2 u-e \sin (1+u)}{2 v} .
$$

In this case, $\mathbf{M}$ is parametrized by

$$
\mathbf{x}(u, v)=\left(\begin{array}{c}
v \cos u \\
v \sin u \\
e^{u}+v\left(\frac{-e^{u}+e \cos (1-u)+2 c_{3} v \cos u+2 c_{4} v \sin u+e^{u} \sin 2 u-e \sin (1+u)}{2 v}\right)
\end{array}\right) .
$$


Theorem 5.2. Let $\mathbf{M}$ be a ruled surface given by (3.3) in $\mathbb{I}_{3}^{1}$. If $\mathbf{M}$ is harmonic or isotropic minimal, then it is congruent to an open part of the surface

$$
\mathbf{x}(u, v)=\left(\begin{array}{c}
v \cos u \\
v \sin u \\
f(u)+v\left(c_{3} \cos u+c_{4} \sin u+\cos u\left(\int_{1}^{u} \frac{f^{\prime \prime}(x) \sin x}{v} d x\right)\right. \\
\left.-\sin u\left(\int_{1}^{u} \frac{f^{\prime \prime}(y) \sin y}{v} d y\right)\right)
\end{array}\right) .
$$

If $\lambda \neq 0$, from (5.6), we have

$$
\left(\frac{f^{\prime \prime}}{v^{2}}+\lambda f\right)+v\left(\frac{\left(g^{\prime \prime}+g\right)}{v^{2}}+\lambda g\right)=0 .
$$

This equations are second order linear differential equations with constant coefficients. We discuss two cases according to constant $\lambda$.

Case 1: $\lambda>0,(5.9)$ can be separated, we obtain

$$
\left(\frac{f^{\prime \prime}}{v^{2}}+\lambda f\right)=0
$$

and

$$
\left(\frac{\left(g^{\prime \prime}+g\right)}{v^{2}}+\lambda g\right)=0
$$

Therefore, we have

$$
\begin{aligned}
& f(u)=c_{1} \cos u v \sqrt{\lambda}+c_{2} \sin u v \sqrt{\lambda}, \\
& g(u)=c_{3} e^{u \sqrt{-\left(1+v^{2} \lambda\right)}}+c_{4} e^{-u \sqrt{-\left(1+v^{2} \lambda\right)}},
\end{aligned}
$$

where $\lambda, c_{i} \neq 0 \in \mathbb{R}$ and $\left(1+v^{2} \lambda\right)<0$. In this case, $\mathbf{M}$ is parametrized by

$$
\mathbf{x}(u, v)=\left(\begin{array}{c}
v \cos u \\
v \sin u \\
\left(c_{1} \cos u v \sqrt{\lambda}+c_{2} \sin u v \sqrt{\lambda}\right)+v\left(c_{3} e^{u \sqrt{-\left(1+v^{2} \lambda\right)}}\right. \\
\left.+c_{4} e^{-u \sqrt{-\left(1+v^{2} \lambda\right)}}\right)
\end{array}\right)
$$

Case 2: Let $\lambda<0$, from (5.9), we obtain

$$
\left(\frac{f^{\prime \prime}}{v^{2}}-\lambda f\right)=0
$$


and

$$
\left(\frac{\left(g^{\prime \prime}+g\right)}{v^{2}}-\lambda g\right)=0
$$

Therefore, we have

$$
\begin{aligned}
& f(u)=c_{1} e^{u v \sqrt{\lambda}}+c_{2} e^{-u v \sqrt{\lambda}} \\
& g(u)=c_{3} e^{u \sqrt{-1+v^{2} \lambda}}+c_{4} e^{-u \sqrt{-1+v^{2} \lambda}}
\end{aligned}
$$

where $\lambda, c_{i} \neq 0 \in \mathbb{R}$ and $\left(-1+v^{2} \lambda\right)>0$. In this case, $\mathbf{M}$ is parametrized by

$$
\mathbf{x}(u, v)=\left(\begin{array}{c}
v \cos u \\
v \sin u \\
\left(c_{1} e^{u v \sqrt{\lambda}}+c_{2} e^{-u v \sqrt{\lambda}}\right)+v\left(c_{3} e^{u \sqrt{-1+v^{2} \lambda}}+c_{4} e^{-u \sqrt{-1+v^{2} \lambda}}\right)
\end{array}\right) .
$$

Theorem 5.3. Let $\mathbf{M}$ be a non harmonic ruled surface given by (3.3) in the three dimensional simply isotropic space $\mathbb{I}_{3}^{1}$. If the surface $\mathbf{M}$ satisfies the condition $\Delta \mathbf{x}_{i}=\lambda_{i} \mathbf{x}_{i}$, where $\lambda_{i} \in \mathbb{R}, i=1,2,3$, then it is congruent to an open part of the surfaces (5.12) and (5.15).

Acknowledgement 1. The second author was supported by Basic Science Research Program through the National Research Foundation of Korea(NRF) funded by the Ministry of Education (2015R1D1A1A01060046).

\section{References}

[1] M. E. Aydin, A generalization of translation surfaces with constant curvature in the isotropic space, J. Geom, 107/3, (2016), 603-615

[2] Z. Hanifi, M. Bekkar and Ch. Baba-Hamed, Translation surfaces in the threedimensional Lorentz-Minkowski Space satisfying $\Delta r_{i}=\lambda_{i} r_{i}$, Int. Journal of Math. Analysis, 4/17, (2010), 797 - 808

[3] C. Baikoussis and L. Verstraelen, On the Gauss map of helicoidal surfaces, Rend. Sem. Math. Messina Ser. II, 2/16, (1993), 31-42

[4] M. Bekkar, Surfaces of revolution in the 3-dimensional Lorentz-Minkowski space satisfying $\Delta x^{i}=\lambda^{i} x^{i}$, Int. J. Contemp. Math. Sciences, 3/24, (2008), 1173-1185

[5] S. M. Choi, On the Gauss map of surfaces of revolution in a 3-dimensional Minkowski space, Tsukuba J. Math., 19/2, (1995), 351-367 
[6] F. Dillen, J. Pas and L. Verstraelen, On the Gauss map of surfaces of revolution, Bull. Inst. Math. Acad. Sinica, 18, (1990), 239-246

[7] F. Dillen, J. Pas and L. Verstraelen, On surfaces of finite type in Euclidean 3-space, Kodai Math. J., 13, (1990), 10-21

[8] O. J. Garay, An extension of Takahashi's theorem, Geom. Dedicata, 34, (1990), $105-112$

[9] I. Kamenarovic, On natural geometry of ruled surfaces in isotropic space $\mathbb{I}_{3}^{1}$, Rad HAZU, Matematičke znanosti, 4, (1985), 45-60

[10] M. K. Karacan, D. W. Yoon and B. Bukcu, Translation surfaces in the three-dimensional simply isotropic space $\mathbb{I}_{3}^{1}$, Int. J. Geom. Methods Mod. Phys., 13 1650088, (2016), 9 pages

[11] H. Pottmann, P. Grohs and N. J. Mitra, Laguerre minimal surfaces, isotropic geometry and linear elasticity, Adv Comput Math, 31, (2009), 391-419

[12] H. Sachs, Isotrope geometrie des raumes, Vieweg Verlag, Braunschweig, 1990

[13] B. Senoussi and M. Bekkar, Helicoidal surfaces with $\Delta^{J} r=A r$ in 3-dimensional Euclidean space, Stud. Univ. Babes-Bolyai Math., 60/3, (2015), 437-448

[14] Z. M. Sipus, Translation surfaces of constant curvatures in a simply isotropic space, Period Math. Hung., 68, (2014), 160-175

[15] Z. M. Sipus and B. Divjak, Mappings of ruled surfaces in simply isotropic space $\mathbb{I}_{3}^{1}$ that preserve the generators, Monatsh. Math., 139, (2003), 235-245

[16] K. Strubecker, Differentialgeometrie des isotropen Raumes III, Flachentheorie, Math. Zeitsch., 48, (1942), 369-427

[17] T. Takahashi, Minimal immersions of Riemannian manifolds, J. Math. Soc. Japan, 18, (1966), 380-385

[18] W. O. Vogel, Regelflächen in isotropen raum, Journal für die reine und angewandte Mathematik, 202, (1959), 169-214

[19] D. W. Yoon, Surfaces of revolution in the three dimensional pseudo-Galilean space, Glasnik Matematicki, 48/68, (2013), 415-428

[20] D. W. Yoon, Some classification of translation surfaces in Galilean 3-Space, Int. Journal of Math. Analysis, 6/28, (2012), 1355-1361

[21] D. W. Yoon and J. W. Lee, Linear weingarten helicoidal surfaces in isotropic space, Symmetry, 8/11, (2016), 126

Murat Kemal Karacan

Faculty of Sciences and Arts

Department of Mathematics

Usak University

1 Eylul Campus

64200

Usak

TURKEY 
E-mail: murat.karacan@usak.edu.tr

Dae Won Yoon

Department of Mathematics Education and RINS

Gyeongsang National University

660-701

Jinju

South Korea

E-mail: dwyoon@gnu.ac.kr

Nural Yuksel

Erciyes University

Faculty of Sciences, Department of Mathematics

38039

Kayseri

TURKEY

E-mail: yukseln@erciyes.edu.tr

Received: 31.10.2016

Accepted: 3.04.2017 